\title{
Multilinguales
}

15 | 2021

Recherches algériennes en sociolinguistique et en littérature : discours scientifique/discours militant, quelles frontières?

\section{L'identité nationale à travers les manuels scolaires de langue française en Algérie. Cas du manuel de première année moyenne}

The national identity through the French language textbooks in Algeria. Case of the textbook of first year middle school

\section{Adila Sahraoui Idriss et Fatima Zohra Chiali Lalaoui}

\section{CpenEdition}

Journals

Édition électronique

URL : https://journals.openedition.org/multilinguales/6358

DOI : 10.4000 /multilinguales. 6358

ISSN : 2335-1853

Éditeur

Université Abderrahmane Mira - Bejaia

Référence électronique

Adila Sahraoui Idriss et Fatima Zohra Chiali Lalaoui, « L'identité nationale à travers les manuels scolaires de langue française en Algérie. Cas du manuel de première année moyenne », Multilinguales [En ligne], 15 | 2021, mis en ligne le 15 juin 2021, consulté le 14 février 2022. URL : http://

journals.openedition.org/multilinguales/6358; DOI : https://doi.org/10.4000/multilinguales.6358

Ce document a été généré automatiquement le 14 février 2022.

\section{(i) $\odot$

Multilinguales est mise à disposition selon les termes de la Licence Creative Commons Attribution Pas d'Utilisation Commerciale - Pas de Modification 4.0 International 


\title{
L'identité nationale à travers les manuels scolaires de langue française en Algérie. Cas du manuel de première année moyenne
}

\author{
The national identity through the French language textbooks in Algeria. Case of
} the textbook of first year middle school

Adila Sahraoui Idriss et Fatima Zohra Chiali Lalaoui

Dans la plupart des pays, notamment en Algérie, la communauté scolaire peut constituer un lieu privilégié pour forger l'identité nationale de l'apprenant comme le souligne (Outaleb, $2011: 13-23$ ). La communauté éducative dans l'ensemble de ses constituants, en l'occurrence: les enseignants, les moyens pédagogiques, les aides didactiques et même les élèves entre eux, participent de près ou de loin au renforcement de l'identité. Il existe dans le processus d'enseignement/apprentissage une multitude " d'outils pédagogiques et supports didactiques ${ }^{1}$ », néanmoins le manuel constitue par excellence l'outil pédagogique le plus usité au sein de la classe, à défaut de supports élaborés par l'enseignement. Il se caractérise par un ensemble varié de textes, d'images et d'activités ayant une visée didactique et pédagogique.

2 Le manuel scolaire représente le terrain où s'expriment les objectifs du système éducatif, qui tente de s'adapter aux besoins impératifs du développement et aux réalités du monde moderne. En conséquence, le statut du manuel l'expose à des changements constants dus aux réformes, qui tentent de rendre plus efficace l'enseignement/apprentissage des apprenants en tant que futurs citoyens et leur permettre d'accéder à des connaissances mais aussi à des valeurs culturelles, morales et idéologiques². Á cet effet, la loi $n^{\circ}$ 08-04 proclame que «l'école algérienne a pour vocation de former un citoyen doté de repères nationaux incontestables, profondément attaché aux valeurs du peuple algérien [...] et en mesure de s'ouvrir sur la civilisation universelle $»^{3}$. 
Conformément à cette directive, le cahier des charges pédagogique précise que l'identité algérienne doit être présente dans les manuels des différentes disciplines.

Sur le plan pédagogique [...] il faut accorder une proportion significative aux composantes du patrimoine culturel algérien, à concurrence de $80 \%[. .$.$] dans les$ sciences sociales et humaines (littérature, histoire, éducation islamique, éducation civique, histoire, géographie, ...), dans divers supports (textes, illustrations, cartes et documents cartographiques...). Le reste est puisé du patrimoine universel ${ }^{4}$.

Nous pouvons déduire que l'objectif du système éducatif aspire à mettre en évidence l'identité nationale, conformément aux principes idéologiques de l'État algérien et aux textes y afférents. Or, une question essentielle s'impose ici : Á quel degré la dimension de l'identité algérienne est omniprésente dans le manuel scolaire de langue française de première année moyenne ( $2^{\text {éme }}$ génération) ? Autrement dit, le manuel en question contribue-t-il à la construction et au renforcement de l'identité nationale de l'apprenant algérien en conformité avec ce qui est stipulé dans le cahier des charges pédagogique?

En réponse à nos questionnements, nous proposons l'hypothèse suivante :

- Étant conçu pour apporter des améliorations et des complémentarités par rapport au précédent, le manuel de FLE de $1^{\text {ère }}$ AM ( $2^{\text {ème }}$ génération) reflète l'identité nationale de l'apprenant algérien conformément aux recommandations du cahier des charges pédagogique.

Le présent article est axé sur les directives du cahier des charges pédagogique et leur mise en application dans le manuel en question. Pour ce faire, nous avons opté pour une approche qualitative se basant sur l'analyse de contenu permettant d'explorer trois composantes pédagogiques essentielles: les thématiques des textes, la nationalité des auteurs, les sources bibliographiques sélectionnées et les images choisies. Nous nous sommes intéressées au cycle moyen car il représente un nouveau stade pour l'apprenant, après un cursus de cinq années au primaire suivies par une période de trois années au secondaire. Il est donc la phase liant deux cycles différents et complémentaires. En outre, l'esprit critique et imaginatif de l'apprenant peut être sollicité davantage à ce niveau, dans le sens où à cet âge ${ }^{5}$, il est plus facile de lui inculquer des valeurs nationales et universelles.

\section{Manuel scolaire : outil pédagogique pour apprendre}

Dans le processus d'enseignement/apprentissage, le manuel scolaire est considéré comme un outil didactique indispensable qui offre à l'apprenant un recueil de connaissances lui permettant de découvrir, apprendre, comprendre et acquérir des savoirs. Il représente aussi pour l'enseignant une référence de base telle une feuille de route pour la gestion des cours, et permet aux parents l'accompagnement et le suivi de leurs enfants dans l'apprentissage (Gérard et Roegiers, 2009: 83-106). Notons aussi, que le curriculum scolaire ${ }^{6}$ est reflété dans le manuel sous forme de textes et d'images: le message iconique accompagne le message linguistique utilisé à des fins pédagogiques. Dans ce sens, (Lebrun, $2007: 14$ ) note que

le manuel est un objet culturel en soi, qui nous renseigne sur la société globale dont il est issu [...] Analyser les divers manuels d'une société donnée, c'est donc tracer un portrait de cette société elle-même et du type d'élève qu'elle entend former. 
Dans ce contexte, il s'agit pour nous de déterminer la place octroyée aux référents identitaires dans le manuel du FLE de $1{ }^{\text {ère }}$ AM (année 2016 - 2017).

\section{Principes du système éducatif algérien}

7 Il convient de souligner qu'à la lecture de la loi n 08-04 du 15 Moharram1429 correspondant au 23 janvier 2008, portant loi d'orientation sur l'éducation nationale, les principes sont édictés comme suit:

renforcer l'identité des élèves en harmonie avec les valeurs et traditions sociales, spirituelles et éthiques issues de l'héritage culturel commun; s'imprégner des valeurs de la citoyenneté et des exigences de la vie en société; apprendre à observer, analyser, raisonner, résoudre des problèmes, développer[...] leur curiosité, leur imagination, leur créativité et leur esprit critique; avoir une ouverture sur les civilisations et les cultures étrangères... (Chapitre III. Art. 45).

Il est bien entendu que ces objectifs sont favorablement recommandées en insistant sur la préservation des composantes principales de l'identité nationale qui peuvent être définies comme étant l'ensemble des mœurs, traditions, rites, langues, croyances et l'histoire qui jalonne la mémoire d'une société :

"l'identité est à la fois personnelle et sociale; elle exprime en même temps la singularité individuelle et l'appartenance à des "catégories sociales" (familiales, locales, ethniques, sociales, idéologiques, religieuses...) » (Costalat-Founeau \& Lipiansky, 2008 : 3). Á cet égard, le Ministère de l'Éducation a adopté une réforme apportant des remaniements au niveau des programmes et manuels scolaires, pour réaliser concrètement les objectifs généraux visés. En effet, la loi du 23 janvier 2008 n 08-04, portant loi d'orientation sur l'éducation nationale, s'inscrit dans une perspective visant une ouverture sur le monde extérieur en vue du développement des échanges entre les civilisations, l'intégration de l'interdisciplinarité et du savoir-faire, l'éducation à la citoyenneté et la valorisation de l'identité nationale (articles 4, 5 et 6).

Ces intentions, sont traduites dans les finalités du cahier des charges pédagogique ministériel et doivent être appliqués dans les supports didactiques (curriculums et manuels scolaires) de toutes les disciplines, touchant en grande partie ceux des langues étrangères, $d u$ fait qu'elles peuvent véhiculer une culture autre que celle de l'apprenant.

Les langues étrangères sont enseignées en tant qu'outil de communication permettant l'accès direct à la pensée universelle en suscitant des interactions fécondes avec les langues et cultures nationales. Elles contribuent à la formation intellectuelle, culturelle et technique et permettent d'élever le niveau de compétitivité dans le monde économique ${ }^{7}$.

\section{Cahier des charges pédagogique ministériel}

10 Il s'agit d'un document pédagogique contractuel qui trace les directives à respecter par les concepteurs de manuels. Il décrit précisément les besoins auxquels le soumissionnaire doit répondre. Ce document est promulgué par le Ministère de l'éducation nationale en partenariat avec l'Institut national de recherche en éducation INRE- et la Commission d'agrément et d'homologation. Il est édité en 2016 et comprend vingt-deux pages incluant quatre parties: les moyens didactiques; les conditions d'admissibilité à l'agrément et à 
l'homologation; les critères d'évaluation et enfin le barème de notation du matériel didactique pour une évaluation concurrentielle.

Dans son ensemble, le cahier des charges pédagogique algérien met l'accent sur l'importance d'introduire l'identité algérienne dans le contenu pédagogique du manuel scolaire. Conformément à la loi d'orientation 08-04 du 23 janvier 2008, les critères d'évaluation retenus dans ce cahier des charges pédagogique s'inspirent :

- des fondements de l'École algérienne, article 2 chapitre 1 ;

- des missions de l'École algérienne, article 4 chapitre $2^{8}$.

En relation avec les autres disciplines, l'enseignement du français, «contribue à la concrétisation des objectifs de transmission et d'intégration des valeurs républicaines et démocratiques, identitaires, sociales et universelles ${ }^{9}$. Cette présente étude s'intéresse particulièrement au manuel de langue française de première année moyenne (deuxième génération), ayant subi des modifications suite à la réforme, et à travers lequel nous allons décrypter la dimension de l'identité nationale de l'apprenant algérien véhiculée via ses espaces textuels et graphiques. Dans cette perspective, nous allons adopter une approche analytique qui « consiste à décomposer l'objet d'étude en allant du plus complexe au plus simple. Cette méthode recherche le plus petit composant possible, l'unité de base des phénomènes" (Aktouf, 1992: 23), ce qui donnera lieu à une distinction des thématiques favorisés dans le manuel; l'identification de la nationalité dominante des auteurs et le choix des illustrations ayant un rapport avec l'identité algérienne.

\section{Identité nationale}

13 Nous rappelons que des travaux existent sur la question

de l'identité nationale ${ }^{10}$ dans les manuels scolaires en tant

que

« principaux transporteurs de valeurs culturelles

et idéologiques » (Abadi, $2013: 137-141$ ).

Les manuels d'histoire soulignent que le

« référent arabo-islamique a besoin d'être articulé à des contextes et cadres sociohistoriques au sein desquels les apprenants sont directement intégrés dans leur vie quotidienne » (Remaoun, 1993 : 57-64).

Ainsi, les manuels d'histoire peuvent façonner

« une image homogène de l'identité à travers la multiplicité des catégories, des classes sociales, des régions et des ethnies ». (Ait Saadi Bouras, 2015 : 445-453).

Entre autres, dans les manuels des sciences islamiques, dits facteurs de l'identité nationale, l'école algérienne se voit imposer une dichotomie entre une formation de

«citoyens d'une société démocratique et des individus religieux pour une communauté religieuse ». (El-Mestari, $2011: 70-80)$.

S'agissant des manuels du FLE, il est signalé

«qu'exclure complètement la culture étrangère ne peut aider les élèves à construire leur identité communautaire et nationale». (Khadir, 2016: 1-16); (Bouari, 2011 :127-132).

La relation langue/culture est étroitement liée partant du principe que « la classe de langue est définie comme un des lieux où la culture du pays de l'élève et la culture étrangère enseignée entrent en relation » (Zarate, 1995 :11). 
Ainsi, l'enseignement du FLE n'échappe pas à la dimension culturelle de la langue en question.

En effet, la langue ne constitue pas uniquement un vecteur de communication, mais également une dimension qui relève de la culture d'un groupe social (Hamidou, 2007 : 29-40). En tant que substance sociale et culturelle, la langue constitue l'identité d'une société. Dans les sciences humaines et sociales, la définition de l'identité demeure équivoque. De ce fait, il n'est pas si évident d'en donner une définition simple et précise. Toutefois, les paradigmes identificatoires comme la langue, la religion, l'histoire, la culture, les traditions et les coutumes sont des liens symboliques permettant de créer d'une part, un socle commun dans une société donnée, et d'autre part assurer des distinctions anthropologiques.

Nous relevons dans le cahier des charges pédagogique

général les mêmes composantes fondamentales de l'identité algérienne signalées dans le préambule de la révision constitutionnelle de la République algérienne démocratique et populaire (2016:3). En termes de constantes nationales, l'école algérienne a pour missions d'" affirmer la personnalité algérienne et consolider l'unité de la nation par la promotion

et la préservation des valeurs en rapport avec l'Islamité, l'Arabité et l'Amazighité [...] $»^{11}$. Ainsi notre étude se focalise sur l'approche de l'identité algérienne, comme ensemble constitué de repères nationaux: religion (l'islam), arabité, amazighité, histoire, nationalité

et patrimoine algérien (traditions, coutumes, habillement, cuisine algérienne, prénoms arabes, etc.).

\section{Présentation du corpus}

17 Le manuel scolaire sélectionné pour notre étude se compose de 174 pages, il est édité et mis en circulation en 2016, conçu par une inspectrice de l'éducation et de l'enseignement moyen ${ }^{12}$ avec la participation de deux professeurs de français ${ }^{13}$. Ce livre se constitue de 3 projets dont chacun comporte une brève présentation et une situation de départ. Ces projets sont divisés à leur tour en séquences présentées comme suit :

- Projet 1 : présente l'introduction suivante: «afin de célébrer les journées mondiales de la propreté et de l'alimentation qui se déroulent les 15 et 16 octobre de chaque année, mes camarades et moi élaborerons une brochure pour expliquer comment vivre sainement ${ }^{14}$. Il comprend trois séquences définit chacune par un intitulé :

- Séquence 1 : j'explique l'importance de se laver correctement.

- Séquence $2:$ j'explique l'importance de manger convenablement.

- Séquence 3 : j'explique l'importance de bouger régulièrement.

- Projet 2 : est introduit par l'intermédiaire de l'énoncé suivant: «je réalise avec mes camarades un dossier documentaire pour expliquer les progrès de la science et leurs conséquences » (Idem., :73). Il se subdivise pareillement en trois séquences :

- Séquence 1 : j’explique les progrès de la science.

- Séquence $2:$ j'explique les différentes pollutions.

- Séquence $3:$ j'explique le dérèglement du climat.

- Projet 3 : comprend une brève introduction : sous le slogan

"pour une vie meilleure », je réalise avec mes camarades un recueil de cosignes pour se 
comporter en éco-citoyen. (Ibid., :135). Contrairement aux deux premiers projets, ce dernier ne comporte que deux séquences intitulées comme suit :

- Séquence 1 : j'incite à l'utilisation des énergies propres.

- Séquence 2 : j'agis pour un comportement éco-citoyen.

\section{Méthodologie adoptée} adopté une démarche nécessitant une lecture attentive, suivie d'une analyse et interprétation de son contenu. Cette démarche "permet de retracer, de quantifier, voire d'évaluer, les idées ou les sujets présents dans un ensemble de documents: corpus " (Leray, 2008:5). Cette opération nous permettra de repérer les différentes thématiques abordées, les auteurs et les sources bibliographiques sélectionnés, ainsi que les illustrations fournies dans le but de vérifier la présence d'indices des référents identitaires algériens. Il est à noter que l'identité en tant que catégorie générale complexe se décline à travers les pratiques langagières, les croyances, le sentiment national, la culture, les coutumes et traditions, etc.

\section{Analyse thématique}

Partant du principe que l'analyse thématique est partie intégrante de l'approche qualitative qui s'insère dans les techniques d'analyse de contenu permettant d'identifier les thèmes des textes (ce dont parle le texte), il nous a semblé pertinent d'opter pour cette démarche. Cette analyse consiste à

«transposer d'un corpus donné en un certain nombre de thèmes représentatifs du contenu analysé ». (Mucchielli, 2012: 238) Cela dit, notre corpus comprend soixantetreize textes qui sont principalement de type informatif, explicatif et prescriptif dont nous allons identifier les thématiques sous format de graphes en secteur.

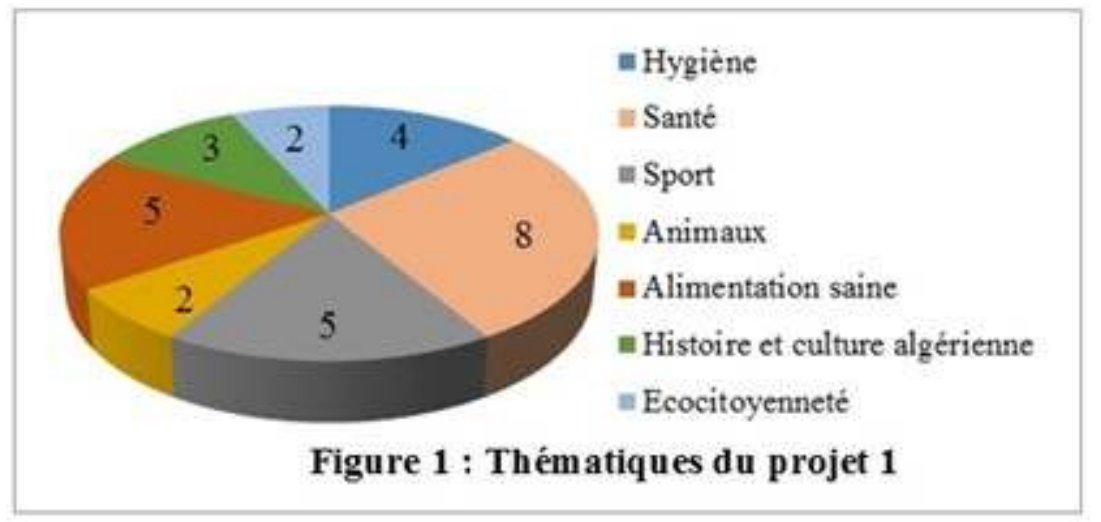

Commentaire : Le projet 1 comprend vingt-neuf textes répartis comme suit : huit qui évoquent le thème de la santé, une égalité de cinq textes pour chacune des deux thématiques, celle de l'alimentation saine et du sport, s'en suit celle de l'hygiène (4).

Nous citons ici à titre d'exemple quelques intitulés: "problème de santé et information » (p.18); « une alimentation saine » (p.44); « l'utilité du sport scolaire » (p. $64)$; « des gestes simples pour une bonne hygiène corporelle » (p.14); etc. Cependant les sept textes restants dont trois conçus pour une lecture récréative sont porteurs de thématiques variées 
entre histoire et culture algérienne, écocitoyenneté et animaux :

«Bleu, blanc, vert» (p. 70); « Si Bachir» (p. 28),

«Le fennec» (p.56), etc. En somme, nous pouvons retenir que le thème principal dominant dans cette partie est celui de la "santé » dans le sens où il englobe les thématiques de l'hygiène, l'alimentation saine et le sport. En ce qui concerne la question de l'identité nationale, elle est très peu évoquée même si nous pouvons y référer de manière superficielle dans les textes suivants :

- $\circ$ «Le tabac tue »: ce texte rapporte particulièrement les statistiques faites en Algérie sur les personnes qui décèdent au quotidien à cause du tabac, et ce, lors d'une journée de sensibilisation organisée au profit de 120 lycéens de la wilaya d'Oran. Toutefois, le thème choisi relève d'un sujet d'ordre universel sur la nocivité du tabac.

- "À quoi servent les UDS »: le texte parle de l'unité de dépistage et de suivi du Ministère de la santé qui contrôle l'hygiène des établissements scolaires en Algérie.

- «Problèmes de santé et information » : un docteur algérien explique le rôle des médecins, des journalistes et des écoles dans la diffusion de l'information concernant les problèmes de santé. Dans ce texte, l'identité algérienne n'est référée que par rapport à la nationalité du docteur.

- «La journée mondiale de l'hygiène des mains »: il s'agit de la participation de l'Algérie à la célébration de cette journée mondiale pour l'amélioration de l'hygiène des mains dans les hôpitaux. Il faut noter ici que la célébration de cette journée est d'ordre mondial (universel), et l'Algérie en fait partie (membre participant).

- «Le terfès » : présente un plat populaire du sud algérien (Sahara).

- "Surpoids et obésité chez les enfants algériens»: étude effectuée sur le surpoids et l'obésité chez les enfants scolarisés à Tébessa (Algérie).

- «Le fennec » : animal adapté à la vie du désert et vit dans le Sahara. Nous signalons ici que cet animal vit aussi au sud algérien (Sahara) et que c'est en rapport à cela qu'on surnomme les joueurs de football algériens « les Fennecs ».

- «La longue marche » : texte rhétorique abordant l'exil qui peut-être référé à la Guerre de libération nationale.

- «Coupe du monde » : présente un résumé sur un match joué par l'Algérie lors de la coupe du monde de 2014.

○ « Noureddine Morceli » : relate les exploits d'un athlète algérien.

21 Résultat (s) : Pour cette partie, nous constatons que sur les vingt-neuf textes du premier projet, nous relevons uniquement dix textes incluant des indices relatifs à l'identité algérienne, à savoir: la nationalité, l'histoire, les personnalités, les noms arabes (Halliche, Djabou, Morceli...) et la cuisine algérienne (terfès). 


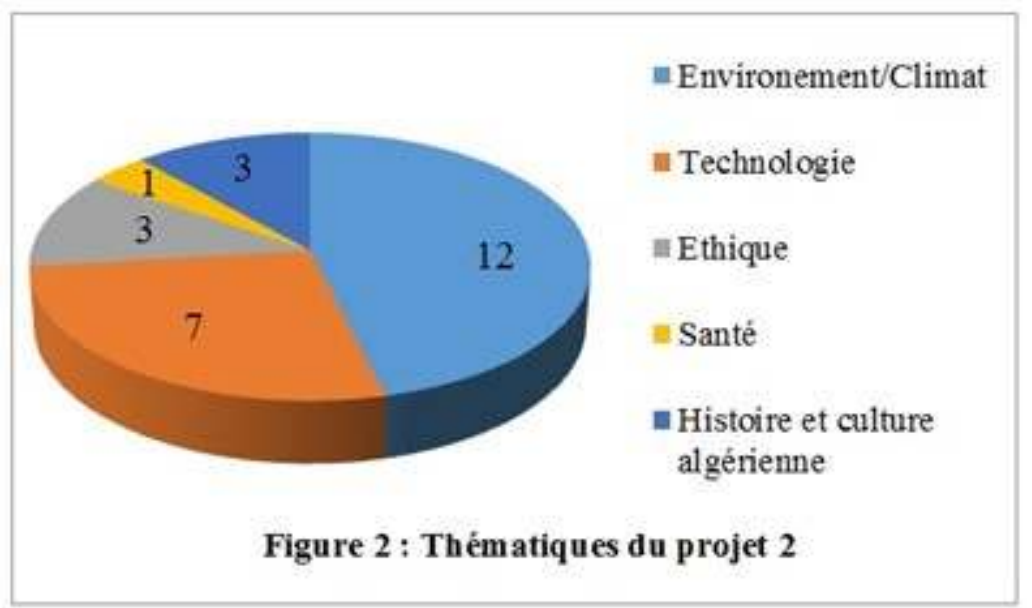

Commentaire : Le projet 2 comporte vingt-sept textes dont douze traitent le thème de l'environnement, sept textes d'ordre technologique, tandis que les sept autres restants dont trois destinés pour une lecture récréative portent des thèmes diversifiés, à savoir, l'éthique (3), l'histoire et culture algérienne (3), ainsi que la santé (1). Nous citons ici quelques exemples: "La couche d'ozone va-t-elle disparaître » (p.106); «Pourquoi Internet a-t-il modifié notre vie ?» (p. 86) ; «Ali le pêcheur» (p.92); «La patrie » (p. 112), etc.

Les données recueillies permettent donc d'identifier deux thématiques prédominantes dans le second projet, celles de l'environnement et de la technologie. Toutefois, nous relevons des symboles de l'identité algérienne dans les textes suivants :

- «Ali le pêcheur» : relate l'histoire d'un pêcheur doté de valeurs humaines te morales, telles, la naïveté, la générosité et l'honnêteté. De ce fait, le fond de l'histoire présentée dans le manuel évoque principalement des valeurs humaines (universelles). Néanmoins, le personnage principal choisi dans le texte porte un prénom arabe (Ali).

- « Ils vont dans la légende »: ce poème exprime le deuil et la tristesse face à la perte des êtres chers lors de la Guerre de libération algérienne.

- «La patrie » : extrait d'un roman évoquant la période coloniale en Algérie.

- «Mon oncle» : l'indice pouvant marquer l'identité algérienne dans cet extrait de roman, est le terme " gandoura », vêtement souvent porté en Algérie,

○ «Qu'est-ce qu'une foggara?»: dans ce texte, l'auteur emploie les termes arabes « oued» et «foggara » et mentionne la région du sud algérien « Sahara ».

- «La sécheresse en Algérie » : cet extrait traite la question de la sècheresse en Algérie.

- «Algérie » : ce poème reflète l'identité algérienne par la mention de plusieurs régions de l'Algérie souffrant de la violence de la guerre.

Résultat (s) : Nous constatons qu'il n'existe que sept textes dans le projet 2 portant des symboles de l'identité algérienne, à savoir: l'histoire, l'habillement, les régions d'Algérie, les termes et prénoms arabes. 


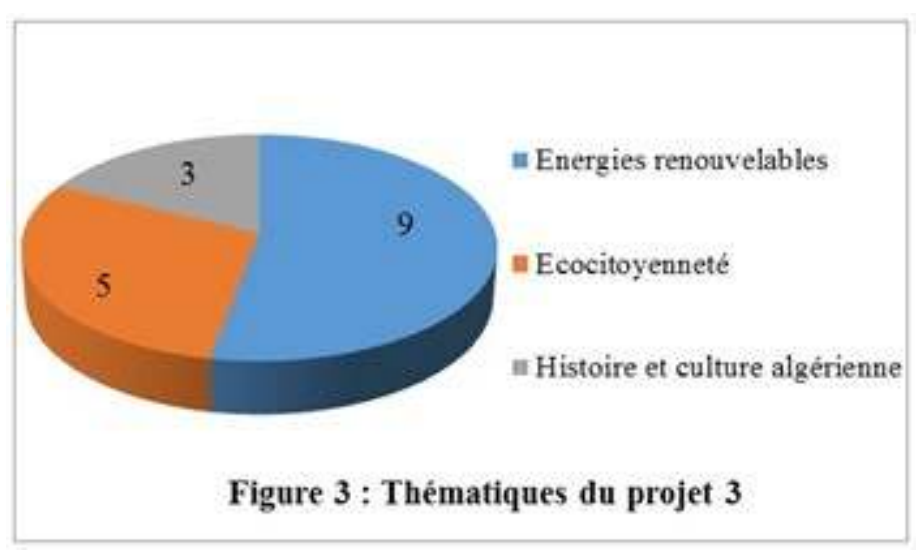

Commentaire : Le projet 3 comprend dix-sept textes dont neuf traitent le sujet des énergies renouvelables, cinq autres, celui de l'écocitoyenneté et les trois restants évoquent l'histoire et la culture algérienne. Á titre d'exemple, voici quelques intitulés : «Pourquoi devrions-nous utiliser des énergies renouvelables?» (p.142); «Les bons réflexes pour devenir un éco-citoyen » (p.160); «Boussoulem» (p. 140), etc. En somme, le projet 3 adopte le thème des énergies renouvelables et de l'écocitoyenneté. Toutefois, l'identité algérienne ne figure superficiellement que dans les textes suivants :

- «Boussoulem » : cet extrait porte sur le patrimoine culturel algérien dans lequel l'auteur relate la beauté de son pays natal la Kabylie et la cuisine traditionnelle connue dans cette région

« couscous, huile d'olive... ».

- "L'Algérie et le développement des énergies propres »: ce texte traite la question des énergies propres dans un contexte algérien. Néanmoins, la thématique ici est d'ordre technologique et non pas identitaire.

- "Les énergies vertes »: cet extrait expose un entretien avec un professeur-chercheur algérien (Mme Benhamou) de l'université de Boumerdés en rapport avec les énergies renouvelables.

- «Les meringues » : cet extrait de roman croise deux civilisations différentes (algérienne et française) reflétées dans les prénoms arabes et français des personnages (Nfissa, Lalla Aïcha, Si Othmane et Jacqueline), ainsi que dans l'ambigüité à comprendre deux termes : " marraine »: femme désignée par les parents dans le but d'accompagner et de soutenir leur enfant, particulièrement en leur absence, culture qui n'est pas accoutumée en Algérie ; et «meringue » : pâtisserie d'origine européenne très connue en France.

- «La citronnade » : ce texte présente une recette algérienne ${ }^{15}$ d'une boisson.

- «Pour une facture d'eau moins salée»: texte portant sur les directives pour la consommation rationnelle de l'eau dans la wilaya d'Oran.

- "De l'électricité pour 20 villages isolés du Sud algérien»: texte informatif sur l'introduction de la filière solaire en vue du développement des énergies renouvelables dans 20 villages du Sud algérien.

- "L'Algérie, $1^{\text {er }}$ pays dans le monde arabe en matière de protection de l'environnement »: extrait tiré du journal El Moudjahid sur une journée d'étude, concernant la protection de l'environnement dirigée par Soumia Abdel Sadok, présidente du Conseil d'Etat algérien.

Résultat (s) : Nous relevons dans le projet 3, huit textes porteurs d'indices de l'identité algérienne tels : des éléments de l'histoire, des prénoms arabes, des régions, cuisine et personnalités algériennes. 


\section{Analyse des résultats de l'approche thématique des trois projets}

Nous constatons que sur un total de soixante-treize textes dans le manuel de FLE de $1^{\text {ère }} \mathrm{AM}$ ( $2^{\text {ème }}$ génération), nous relevons vingt-cinq textes, équivaut à $34.25 \%$ incluant des facteurs reflétant l'identité nationale. Il faut noter ici, que certains extraits choisis dans le manuel appartenant à de grands auteurs algériens comme Yasmina Khadra, Assia Djebar, Tahar Ouettar, Mouloud Féraoun, etc. qui traitent souvent du thème de la Guerre de libération algérienne n'abordent pas explicitement les éléments de l'identité algérienne. L'analyse thématique du manuel en question, nous amène à déduire que la majorité des thèmes abordés s'imprègnent principalement du contexte d'actualité qui relève d'une politique universelle (santé, hygiène, environnement, technologie, écocitoyenneté, etc.). Cependant, le facteur identitaire national est négligé dans les thématiques proposées dans notre corpus.

Notre recherche montre, en conséquence, que le corpus étudié ne reflète pas les recommandations du cahier des charges pédagogique, qui favorise la prise en charge des valeurs identitaires et nationales, dans « le fait de comprendre/dire des énoncés relatifs à un événement national historique, religieux, sportif ou culturel ; de lire/ écrire des textes dont les thèmes sont porteurs de ces valeurs évoquant le patrimoine, les symboles, les traditions, etc. $»^{16}$.

\section{Nationalité des auteurs et références bibliographiques}

Les auteurs sont généralement des témoins de leur époque, tributaires de leur société, faisant référence à leur espace de vie et de pensées. Ils «dressent des tableaux de leur propre société tout en valorisant leur identité et appartenance culturelle» (Boutaghane, 2015: 27). Dans l'intention d'identifier la nationalité culminante des auteurs ainsi que l'origine des sources bibliographiques choisies dans notre corpus, nous allons dresser un tableau pour la constatation des chiffres obtenus.

Tableau 1 : sources bibliographiques et nationalité des auteurs du manuel

\begin{tabular}{|c|c|c|c|}
\hline Auteurs & Nombre & Sources & Nombre \\
\hline \multicolumn{4}{|c|}{ PROJET 1} \\
\hline inconnus & 16 & inconnues & 00 \\
\hline algériens & 08 & algériennes & 14 \\
\hline étrangers & $\begin{array}{l}02 \text { français ; } 03 \text { anglais ; } \\
01 \text { belge ; } 01 \text { danois }\end{array}$ & étrangères & 15 \\
\hline \multicolumn{4}{|c|}{ PROJET 2 } \\
\hline inconnus & 15 & inconnues & 01 \\
\hline algériens & 06 & algériennes & 07 \\
\hline étrangers & $\begin{array}{l}02 \text { français; } 03 \text { anglais ; } \\
01 \text { danois; } 01 \text { belge }\end{array}$ & étrangères & 19 \\
\hline \multicolumn{4}{|c|}{ PROJET 3} \\
\hline inconnus & 12 & inconnues & 02 \\
\hline algériens & 03 & algériennes & 09 \\
\hline êtrangers & 02 anglais ; 01 belge & étrangères & 06 \\
\hline
\end{tabular}




\section{Analyse des résultats relatifs aux auteurs et sources bibliographiques dans le manuel}

Á l'issu des résultats obtenus, nous pouvons conclure que sur une totalité de soixantetreize textes inclus dans notre corpus, un nombre significatif de quarante-trois textes ne fournit pas d'informations sur l'auteur. Néanmoins, ceux dont les auteurs sont mentionnés, se répartissent équitablement entre auteurs de nationalité algérienne (17) tels Assia Djebar, Mohamed Dib, Mouloud Feraoun, Abdelhamid Benhadouga, etc. et ceux de nationalité étrangère (17), notamment française comme Marc Dufumier, Philipe Corentin, Cathy Franco, etc.

Á propos des textes qui ne mentionnent pas d'auteurs, ils sont tirés de différentes sources (site internet, journal, encyclopédie, etc.). Cependant, il faut noter que les sources étrangères (40) sont dominantes par rapport à celles algériennes (30). Ceci nous laisse dire que les directives adressées aux auteurs-concepteurs de manuels scolaires ne concordent pas avec les données recueillis à travers notre analyse. En effet, en matière de représentation des valeurs nationales et universelles, le matériel didactique doit selon le cahier des charges :

- [...] intégrer des textes authentiques, extraits d'œuvres littéraires d'auteurs algériens, tous genres confondus (roman, nouvelles, poèmes, pièces de théâtre, etc.) et du patrimoine culturel national (contes, légendes, mythes, etc.), à concurrence de $80 \%$;

- intégrer des textes littéraires du patrimoine universel, à concurrence de $20 \%$. Pour les autres langues étrangères (Langue étrangère 2), intégrer des textes d'auteurs algériens, autant que possible ${ }^{17}$.

\section{Les illustrations du manuel et leurs corrélations avec l'identité algérienne}

Les documents iconographiques qui désignent l'ensemble des images représentant un dessin, une carte, un graphe, une photographie, etc. servent à expliquer, décrire et donner une idée plus claire. À ce propos, (Biron, 2006 :196) signale la

"nécessité d'introduire l'image pour rendre concret ce qui est abstrait». L'image permet donc d'illustrer et d'expliquer le code linguistique. Notre corpus présente une abondance d'illustrations regroupant en grande partie des photographies et des dessins de bonne résolution. Bien entendu, environ 80 images occupent une surface très importante dans les pages du manuel.

Par ailleurs, les illustrations sélectionnées sont d'ordre universel, mettant en scène des photos et des dessins reflétant l'hygiène, la nourriture, le sport, la technologie, l'environnement, etc. Une autre minorité (13 images) représente principalement des personnalités algériennes et des lieux appartenant au territoire national :

- $\circ$ la place de l'Emir Abdelkader (Alger, Algérie); un village au sud algérien (Sahara) et un village en Kabylie (Algérie);

- auteurs algériens: Abdelhamid Benhadouga, Mouloud Mammeri, Maïssa Bey, Tahar Ouettar, Mohamed Dib et Assia Djebar ;

- athlètes algériens : Noureddine Morceli et Hassiba Boulmerka. 
- La dominance des images d'ordre scientifique et universel laisse à supposer que les éditeurs du manuel tentent de concilier avec les objectifs de la politique éducative avec la volonté de s'intégrer à un monde en constante progression.

31 Par l'entremise de notre étude, nous cherchions à déterminer si l'école algérienne véhicule via ses manuels une représentation assez large de l'identité algérienne, ou uniquement une image imprécise de celle-ci. Cependant, à travers la lecture du manuel

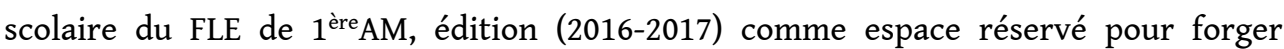
l'identité algérienne, nous pouvons constater que les référents qui se rapportent à cette identité sont négligés dans l'ensemble. En effet, comme nous l'avons pu remarquer via l'analyse effectuée, nous retenons que :

- $\circ$ les thématiques reflétant l'identité algérienne ne représentent que $34 \%$ de la totalité des textes (73) proposés dans notre corpus. Nous pouvons relever le thème de la guerre de libération nationale ainsi que la présence de quelques indices pouvant refléter l'identité nationale, tels : les termes et noms arabes, cuisine traditionnelle algérienne, régions de l'Algérie, etc.

- les auteurs sont sélectionnés de manière équitable entre nationalité algérienne et étrangère, notamment française avec un pourcentage significatif de $50 \%$.

- l'iconographie occupe une grande place dans les pages du manuel à raison de 80 images. Toutefois, celles qui ont proprement représenté l'Algérie sont à proportion de $16 \%$, et ce, à travers la référence des personnalités et régions algériennes.

Il est concevable que le contenu didactique et pédagogique couronne l'action de modernisation et la formation identitaire du citoyen. Attitude clairement constatée dans le manuel en question qui s'appuie de manière globale sur une sélection de textes d'ordre universel qui coïncide probablement avec l'intention politique éducative du pays désirant répondre aux besoins impératifs de la mondialisation. Toutefois, on soulève une certaine marginalisation vis-à-vis des indices pouvant renvoyer à l'identité algérienne comme il est censé être établi selon les directives du cahier des charges pédagogique relative à la langue française pour le cycle moyen.

\section{BIBLIOGRAPHIE}

ABADI, D. (2013). «L'image de l'identité nationale en contexte didactique algérien. Cas des manuels scolaires du FLE », Al Athar, 18, vol. 12, 11-14.

AIT SAADI BOURAS, L. (2015). L'histoire nationale algérienne à travers ses manuels scolaires d'histoire, 445-453. Disponible sur :

[https://books.openedition.org/enseditions/2417 ?lang =fr] (consulté le 10/09/2019).

AKTOUF, O. (1987). Méthodologie des sciences sociales et approche qualitative des organisations. Québec : PUQ. 
BIRON Diane, «Fonctions de l'image dans les manuels scolaires de mathématiques au primaire », dans Lebrun Monique (dir.), (2006). Le manuel scolaire : un outil à multiples facettes. Québec : Presses de l'Université du Québec.

BOUARI, H. (2011). Le manuel de français de 5AP : Quels traits identitaires et quelles représentations culturelles y véhiculés? In Actes du Séminaire national : Enseignement/ apprentissage y-sont français en Algérie : Enjeux culturels et représentations identitaires, 127-132.

Bulletin officiel de de l'éducation nationale (2008). Loi d'orientation sur l'éducation nationale $\mathrm{n}^{\circ}$ 08-04 du 23 janvier 2008, article 45.

CALABRE, I. (1988). « Le rôle de l'iconographie », Revue des livres pour enfants, 122-123, Bibliothèque nationale de France, 1988, 84-91.

CALINDERE, O-C. (2010). L'identité nationale et l'enseignement de l'histoire. Analyse comparée des contributions scolaires à la construction de l'identité nationale en France et Roumanie (1950-2005), Thèse pour le Doctorat en Science politique, Université de Bucarest, Bucarest, Roumanie.

Conseil national des programmes (2009). Référentiel général des programmes.

COSTALAT-FOUNEAU, A-M. \& LIPIANSKY, E-M. (2008/1). Éditorial. « Le sujet retrouvé ». In Connexions, Cairn. info, 89, Belgique, 2008/1, 7-12.

DEMOUGIN, F. \& SAUVAGE, J., « Construction identitaire à l'école », Tréma, 33-34, Faculté d'éducation de l'université de Montpelier, 2010, 1-8.

DJABI, N. \& BOUKROUH, N., (2015) Nabni. Débats Algérie rêvée sur l'identité et les langues : comment renouveler le récit national ? Disponible sur : [https://www.youtube.com/watch? v =e1_ICp2H-vg\&t =2978s] (consulté le 03/08/2019).

EL-MESTARI, D., « Le discours religieux des manuels scolaires algériens de l'éducation islamique dans le cycle secondaire ", Tréma, 35-36, Faculté d'éducation de l'université de Montpelier, 2011, 70-80.

GERARD, F-M. \& ROEGIERS, X., (2009/2). Des manuels scolaires pour apprendre : concevoir, évaluer, utiliser. Bruxelles : De Boeck supérieur. (83-106)

HAMIDOU, N. « La langue et la culture : une relation dyadique », Synergies Algérie, 1, France : Gerflint, 2007, 29 - 40.

KHADIR, S. (2016). « Étude socioculturelle du manuel scolaire de FLE en Algérie. Cas du manuel de première année moyenne », Dirassat \& Abhath, 24, 1-16.

LERAY, Ch. (2008). L'analyse de contenu : de la théorie à la pratique. Québec : Presses de l’Université du Québec.

LOUBET, J.L, (2000). Initiation aux méthodes des sciences sociales. Paris : L'Harmattan.

MERAGA, C., BOUZELBOUDJEN, H. \& MADAGH, A., (Eds.), Mon livre de langue française, 1 ère année moyenne, (2017-2018). Réghaïa, Alger : ENAG Éditions.

Ministère de l'Éducation nationale, Institut nationale de recherche en éducation \& Commission d'agrément et d'homologation, Cahier des charges pédagogique général, Discipline : Français, palier du cycle moyen, 2016, 
Ministère de l'éducation nationale, Commission nationale des programmes, Groupe spécialisé disciplinaire du français (2016). Document d'accompagnement du programme de français -cycle moyen-.

OUTALEB, A., (2011). « Les connaissances culturelles au service de la compétence communicative. L'enseignement du FLE en Algérie ». In Actes du séminaire national : enseignement/ apprentissage du français en Algérie : enjeux culturels et représentations identitaires, 13-23.

PAILLE, P. \& MUCCHIELLI, A., (2012). L'analyse qualitative en sciences humaines et sociales, Paris : Armand Colin.

Présidence de la République \& secrétariat général du gouvernement (2016). Constitution de la République algérienne démocratique et populaire. Loi n 16-01 du 6 mars 2016, Journal officiel $\mathrm{n}^{\circ} 14 \mathrm{du} 7$ mars 2016.

REMAOUN, H., (1993/2). « Sur l'enseignement de l'histoire en Algérie ou de la crise identitaire à travers (et par) l'école », NAQD, 5, 57 - 64 .

ZARATE, G., (1995), Représentations de l'étranger et didactique des langues, Paris : Didier.

\section{NOTES}

1. Autrement dit: tout dispositif matériel accompagnant une situation d'enseignement/ apprentissage, tel que, le manuel scolaire, l'ordinateur, le tableau, le $\mathrm{CD}$, le vidéoprojecteur, etc. Un support devient un outil pédagogique lorsqu'il est élaboré pour aider un public à atteindre un objectif dans un contexte donné; il sert en quelque sorte de médiateur, de facilitateur pour apprendre.

2. La loi d'orientation sur l'Éducation Nationale n 08-04 du 23 janvier 2008 précise dans son préambule, notamment dans les chapitres I et II du titre premier et dans les chapitres II, III et IV du titre trois, les missions de l'école en matière de valeurs spirituelles et citoyennes.

3. La loi nº8-04 du 23 janvier 2008 portant loi d'orientation sur l'Éducation Nationale, chapitre I, article 2

4. Ministère de l'Éducation Nationale, l'Institut National de Recherche en Éducation \& la Commission d'Agrément et d'Homologation (2016). Cahier des charges pédagogique général algérien, p. 4.

5. L'OMS considère que l'adolescence est la période de croissance et de développement humain. Il s'agit de l'acquisition de compétences,

la construction et l'exploration identitaire, le choix de valeurs, la création

de l'estime de soi ainsi que la capacité de raisonnement. https://www.who.int/ maternal_child_adolescent/topics/adolescence/dev/fr/

6. Le curriculum est entendu comme le programme scolaire dans son sens le plus large; autrement dit, les objectifs d'enseignement, les objectifs d'apprentissage, les méthodes et démarches, les supports ainsi que l'évaluation.

7. Commission Nationale des Programmes (2009). Référentiel général des programmes, mise en conformité avec la loi nº 08-04 du 23 janvier 2008 p.p.65-66.

8. Ministère de l'Éducation Nationale; l'Institut National de Recherche en Éducation \& la Commission d'Agrément et d'Homologation (2016). Cahier des charges pédagogique général algérien, p. 14.

9. Ministère de l'Éducation Nationale (2016). Document d'accompagnement du programme de français pour le cycle moyen, p. 2 . 
10. La notion de l'identité nationale est complexe et polysémique, elle est sujette à des tensions et discussions idéologiques et politiques, mais comme notre recherche ne s'insère pas directement dans cela, nous nous sommes contentées de donner les grandes lignes de l'identité nationale.

11. Ministère de l'Éducation Nationale, l'Institut National de Recherche en Éducation \& la Commission d'Agrément et d'Homologation (2016). Cahier des charges pédagogique général algérien, p. 3.

12. Anissa Madagh.

13. Chafik Meraga \& Halim Bouzelboudjen.

14. Meraga, C., Bouzelboudjen, H., Madagh, A. (2017-2018). Mon livre de langue française, $1^{\text {ère }}$ année moyenne, Réghaïa, Alger : ENAG Éditions, p. 8.

15. Dans le contenu du texte, il est précisé qu'il s'agit d'une recette algérienne : " pour obtenir une bonne citronnade algérienne, il faut [...]».

16. Ministère de l'Éducation Nationale (2016). Document d'accompagnement du programme de français pour le cycle moyen, p. 5.

17. Ministère de l'Éducation Nationale; l'Institut National de Recherche en Éducation \& la Commission d'Agrément et d'Homologation (2016). Cahier des charges pédagogique général algérien, p. 15.

\section{RÉSUMÉS}

Par le biais de ses textes, ses illustrations et ses activités variées, le manuel scolaire est susceptible de contribuer au renforcement de l'identité algérienne des apprenants. La question est de savoir comment? Notre contribution vise l'analyse des finalités actuelles du système éducatif algérien, traduites en objectifs de formation, via le manuel. Notre corpus est basé sur le manuel de langue française de première année moyenne (édition 2016-2017), dont nous avons fait l'analyse de contenu. À l'issue de notre recherche, il s'avère que ce support pédagogique ne semble pas interférer dans l'identité « nationale » algérienne.

Through its texts, illustrations and varied activities, the textbook can contribute to the strengthening of the Algerian identity within learners. Yet, the question to be asked is "how". This paper aims to analyse the current purposes of the Algerian education system; i.e. the educational objectives stated in the textbook. The corpus of this study is based on the French language textbook of the first year in middle school (2016-2017 edition), whose content is the core of the analysis. From this research, it can be revealed that this pedagogical support does not seem to interfere in the "national" Algerian identity.

\section{INDEX}

Keywords : textbook, French language, national identity, Algerian education system, middle school

Mots-clés : manuel scolaire, langue française, identité algérienne, système éducatif algérien, cycle du moyen 


\section{AUTEURS}

\section{ADILA SAHRAOUI IDRISS}

Université Oran 2, Mohamed Ben Ahmed, Algérie

FATIMA ZOHRA CHIALI LALAOUI

Université Oran 2, Mohamed Ben Ahmed, Algérie 